\title{
El proceso de certificación de calidad para el sector cooperativo: caso de Bogotá y Medellín ${ }^{1}$
}

\author{
Fecha de recepción: 26 de octubre de 2010 \\ Fecha de aprobación: 28 de junio de 2011
}

\author{
Angélica Ausana Castellanos Villamil \\ susanitacas@hotmail.com \\ Universidad Nacional de Colombia \\ Contadora pública, magíster en Administración, \\ Universidad Nacional de Colombia.
}

\section{Alexandra Montoya Restrepo}

lamontoyar@unal.edu.co

Universidad Nacional de Colombia

$\mathrm{PhD}$, administradora de empresas y profesor aso-

ciado de la Facultad de Ciencias Económicas.

\section{Resumen}

En la actualidad, toda empresa necesita ser competitiva y tener altos niveles de productividad. Dentro de esta dinámica también deben estar las organizaciones del sector solidario y, entre éstas, las cooperativas.

Por tal motivo, se requiere contar en la organización con procesos permanentes, continuos y oportunos de investigación, planeación y gestión que permitan cumplir la misión y la visión de las organizaciones solidarias. En este sentido, los sistemas de gestión de la calidad pueden ayudar a las organizaciones a satisfacer las necesidades y las expectativas de las partes interesadas (empleados, asociados, afiliados, beneficiarios, voluntarios y miembros, entre otros) y sus grupos de interés.

Es así como las Normas Iso 9000 se han elaborado para asistir a las organizaciones en la imple-

\author{
Iván Montoya Restrepo \\ iamontoyar@unal.edu.co \\ Universidad Nacional de Colombia \\ Magíster en Administración y profesor asociado \\ de la Facultad de Ciencias Económicas.
}

\begin{abstract}
Today, every company needs to be competitive and have high levels of productivity. Today, every company needs to be competitive and have high levels of productivity. Organizations of the supportive sector, among which there are cooperatives, must also be within this dynamics.

For this reason, it is necessary to have permanent, continuous, and appropriate processes of investigation, planning, and management in order to fulfill the mission and vision of solidarity organizations. In this sense, quality management systems can help organizations to satisfy the needs and expectations of the interested parties (employees, partners, affiliates, beneficiaries, volunteers, and members, among others) and their groups of interest.

Thus, the ISO 9000 family of Standards has been developed in order to help organizations in the im-
\end{abstract}

1 Investigación realizada como tesis de profundización en el marco de la Maestría en Administración de Empresas de la Universidad Nacional de Colombia. 
mentación y la operación de sistemas eficaces de gestión de la calidad. Sin embargo, debido a las circunstancias específicas de las cooperativas, es importante reconocer que no existe una manera única de implementar un sistema de gestión de la calidad basado en la Norma.

En este documento se presentan los resultados de un estudio de investigación realizado a cooperativas en Bogotá y Medellín cuyas experiencias permiten analizar el impacto que tuvo en ellas obtener las certificaciones de calidad, a fin de lograr competitividad en el mercado.

\section{Palabras clave}

Cooperativas, certificación de calidad, Bogotá, Medellín. plementation and operation of efficient quality management systems. However, due to the specific circumstances of cooperatives, it is important to recognize that there is not a single way to implement a quality management system of quality management, based on the Standard.

This paper presents the results of an investigation study carried out in cooperatives located in Bogotá and Medellín, whose experience allows us to study the impact they had when obtaining quality certifications in order to achieve competitiveness in the market.

\section{Keywords}

Cooperatives, Quality Certificate, Bogota, Medellin. 


\section{Introducción}

En Colombia existen aproximadamente siete mil empresas pertenecientes al sector solidario, cuyo objetivo es repartir, invertir y redistribuir los recursos entre sus asociados. Trabajan sin ánimo de lucro e invierten dichos recursos y sus excedentes en mejoras de sus mismas instituciones, con el único fin de prestar un mejor servicio a los usuarios. El grupo cooperativo se encuentra integrado por las Cooperativas de Trabajo (CTA), las del sector real y las de ahorro y crédito. Las cooperativas de trabajo asociado son empresas que vinculan el trabajo personal de sus asociados y sus aportes económicos para la producción de bienes, ejecución de obras o la prestación de servicios en forma autogestionaria; se constituyen con un mínimo de 10 asociados. Dentro de este sector, las cooperativas tienen un gran peso, junto con los Fondos de Empleados y las Asociaciones Mutuales. En Colombia el 78\% de las entidades de economía solidaria son cooperativas. La Confederación de Cooperativas (CONFECOOP) registra un balance de 7.833 cooperativas en el país, con un crecimiento del $11,27 \%$, teniendo presencia en treinta y un departamentos del país. Las empresas son en su mayoría micro, pequeñas y medianas, pero las grandes, que corresponden tan sólo al 0,40\%, manejan el 40,90\% del total de los activos del sector (Revista Poder, 2009).

El sector cooperativo ha sido fundamental en los últimos ańos para el crecimiento de la economía en Colombia. Reúne a 4,50 millones de afiliados y representa una participación del 4,80\% del PIB nacional, generando a su vez 700.000 puestos de trabajo. Este éxito se debe, en gran medida, al empuje del sector financiero cooperativo, que superó la cartera del sector financiero tradicional, demostrando un crecimiento del 2,00\%, según lo reportado en el Encuentro Empresarial Cooperativo organizado en Bogotá por CONFECOOP a finales de 2009.

Las cooperativas tienen cada día mayor participación en distintas actividades productivas. Una de las razones económicas esenciales que se emprenden bajo un esquema cooperativo justifica la utilización del mismo para aprovechar las economías de escala que se logren en la producción, comercialización o prestación de servicios realizados de manera asociada. Al ser entidades sin ánimo de lucro, la generación de excedentes y la inexistencia de pérdidas son la principal base de su subsistencia en el medio empresarial.

Como todos los sectores, el solidario debe enfrentarse a los constantes cambios generados por el entorno e interactuar y competir con empresas organizadas bajo otros esquemas jurídicos. Este escenario lo obliga a operar de manera eficiente y rentable en el mercado, por lo cual debe generar al interior de cada organización herramientas que le permitan ser competitivas. Una de ellas es el sistema de gestión de calidad, que facilita la medición de la satisfacción de los clientes y permite mejoras en los diferentes procesos.

En este documento se busca analizar la incidencia de los procesos de gestión de calidad como herramienta, específicamente para el caso del sector cooperativo. Con el fin de contextualizar a los lectores, inicialmente se presentará un marco teórico con información acerca del estado de la economía solidaria del sector cooperativo, para luego desarrollar la metodología de análisis 
que permitirá medir el beneficio de las certificaciones de calidad en la competitividad de las empresas cooperativas, focalizado en los mercados de Bogotá y Medellín, ya que en estas ciudades se encuentra la mayor concentración de éstas. Finalmente, se darán a conocer las conclusiones derivadas de esta investigación, buscando dar un aporte que permita hacer del sistema de gestión de calidad un parámetro de mejoramiento en las cooperativas.

\section{Presentación del problema}

En la actualidad, el sector de la economía solidaría es reconocido por su solidez y por la confianza que genera entre sus asociados. Está compuesto por 7.141 entidades, de las cuales 5.288 son cooperativas, alrededor del 74,00\%; 1.674 son fondos de empleados, correspondiente al 23,40\%; y 176 , asociaciones mutuales, que representan el 2,50\% (CONFECOOP, 2005).

El sector cooperativo se destaca por su gran participación dentro de la economía solidaria, realizando un importante aporte en términos de bienestar tanto para sus asociados, como para la comunidad en general. La responsabilidad social empresarial es una práctica que ha hecho parte fundamental del cooperativismo, gran parte de su esencia está en la generación de beneficios bajo principios de equidad y cooperación.

Sus activos ascienden a 12,1 billones de pesos, con ingresos equivalentes al 5,05\% del PIв nacional, asociando alrededor de 3,2 millones de colombianos y generando cerca de 94.000 empleos directos y 353.200 bajo la modalidad del trabajo asociado (EL Tiempo, 28 de abril de 2006). Además de ser un modelo económico, se considera como una propuesta que no sólo impacta a los asociados, sino también al país entero, debido a su finalidad de tipo social.

Por los constantes cambios del entorno y las exigencias de la globalización, el sector cooperativo se enfrenta a situaciones (desempleo, pobreza, analfabetismo, entre otros) ante las cuales requiere acciones que le permitan responder y consolidarse como instrumento de desarrollo económico y social. Para esto se deben promover mecanismos de representación, supervisión efectiva, inversiones en tecnología, modernización de las estructuras cooperativas y fomento cooperativo, que permitan mantener un comportamiento creciente y eficiente. En este sentido, se hace importante buscar implementar un sistema de gestión de calidad que facilite la normalización de procesos y el cumplimiento con los estándares nacionales e internacionales.

Finalmente, es importante conocer y estudiar la información en cuanto a la normatividad, requisitos y acciones emprendidos por las cooperativas que han involucrado procesos de certificación de calidad, a fin de determinar si dicha certificación es una herramienta que garantiza la competitividad de las cooperativas o si es un cumplimiento formal requerido que no genera un diferencial en el mercado, especialmente en el caso de las ciudades de Bogotá y Medellín.

Por lo anterior, el objeto de estudio se centrará en la incidencia de involucrar procesos de certificación de calidad de las cooperativas pertenecientes a las ciudades de Bogotá y Medellín, para lo cual se observará las motivaciones de las empresas cooperativas para certificarse en calidad con el fin de analizar los 
beneficios que obtuvieron al implementar el sistema de calidad.

\section{La economía solidaria}

Aproximadamente desde hace unos veinte años, en el mundo se viene promulgando el concepto de economía solidaria, que incorpora racionalidades de cuño solidario, actividades de recursos de la sociedad civil y sectores populares como eje central de los comportamientos económicos. Se identifica por ser un modelo alternativo de y para las mayorías populares, que se fundamenta en su esfuerzo y solidaridad con el objetivo de resolver problemas de tipo ambiental, económico, de pobreza y de exclusión social.

La economía solidaria se puede definir como el sistema socioeconómico, cultural y ambiental desarrollado en forma individual o colectiva a través de prácticas solidarias, participativas, humanistas y sin ánimo de lucro para el desarrollo del ser humano como fin de la economía (Retolaza, Mugarra \& Enciso, 2004). Se caracteriza principalmente por la igualdad, el empleo para las personas desfavorecidas, la cooperación, la falta de ánimo de lucro y el compromiso con el entorno.

Según la ley 454 de 1998, se denomina economía solidaria al sistema socioeconómico, cultural y ambiental conformado por el conjunto de fuerzas sociales organizadas en formas asociativas identificadas por prácticas autogestionarias solidarias, democráticas, humanistas y sin ánimo de lucro, para el desarrollo integral del ser humano como sujeto, actor y fin de la economía.

Dentro de los objetivos de la economía solidaria está, a corto plazo, tener resueltas las necesidades básicas: empleo, ingresos, viviendas dignas, acceso a educación, salud, seguridad, salubridad, etc.; y a mediano plazo, consolidar los objetivos de corto plazo y crear redes de economía solidaria, promover la equidad de género, procurar la sostenibilidad ambiental y crear una institucionalidad requerida.

Los primeros estudios sobre el origen de la economía solidaria en América Latina se deben al chileno Luis Razeto, con su obra Economía popular de solidaridad: Identidad y proyecto en una visión integradora (1986), que tuvo especial cabida en el ámbito de las organizaciones económicas populares, así como en diversas instancias de la Iglesia Católica del continente.

Como explica el autor en su obra, "Para la comprensión de este proceso social hay que partir de sus orígenes, que se remontan en Chile a pocas semanas después del pronunciamiento militar de 1973. [...] aquellos acontecimientos significaron una verdadera ruptura en la historia del movimiento social y de organización popular, aún más radical y drástica que la ruptura que implicaron a nivel de las instituciones estatales y de los procesos económicos y políticos nacionales. Ambas rupturas están estrechamente vinculadas y son constitutivas de una misma nueva situación práctica” (Razeto, 1986, p. 14).

Otra fuente importante para hablar del surgimiento de la economía solidaria en Latinoamérica lo constituyen los escritos del entorno de la Confederación Latinoamericana de Cooperativas de Trabajadores (COLACOT), con sede en Colombia. El mérito de la COlACOT reside en haber divulgado estas temáticas en ambientes cooperativistas y laborales 
(COLACOT es un organismo funcional de la Central Latinoamericana de Trabajadores [CLAT] que optó en su x Congreso por la construcción de una economía de la solidaridad en su estrategia de desarrollo) y haber realizado, desde fines de los ochenta, numerosos encuentros que versaban sobre dicha materia. Salta a la vista, en tal sentido, una intención más militante que analítica en estas materias, más allá de esfuerzos como el de la elaboración en 1997 de un ambicioso programa de planificación macroeconómica de largo plazo, conducente a llevar al sector solidario de la economía desde una incidencia del 5,2\% del PBI, hacia el $33,6 \%$ en ocho años (www.economiasolidaria.org).

La tercera fuente proviene de Brasil. A mediados de los años noventa comienza a divulgarse el concepto de la economía de la solidaridad por parte de algunas ONGS y diversas organizaciones populares e incluso sindicales. Sus aportes más relevantes son la crítica que hacen de las estructuras económicas contemporáneas y el rescate de la autogestión y el asociacionismo en las clases populares. En ese sentido, la economía de la solidaridad adquiere características más radicales que las que se encuentran en otros contextos y, por lo general, con un discurso marcadamente más político.

En Europa el desarrollo del concepto economía de solidaridad comienza a manejarse a finales de los años ochenta bajo un aspecto teórico difundido por los académicos y uno práctico divulgado por las ONGs. A nivel teórico, el aporte más importante se debe a Jean Louis Laville. Desde posturas más centralistas hasta otras más comunitarias, este sociólogo francés hace referencia a la importancia que tuvo el siglo XIX en la materia, admitiendo diversos principios y prácticas distintas a las del mercado. "A pesar de las diferencias, dichas prácticas comparten características que permiten establecer paralelismos: todas ellas intentan introducir la noción de solidaridad en las actividades económicas, abogando de esta manera por una economía solidaria" (Laville J.L., 1994, p. 21).

Otros autores, también de Francia, circunscriben la economía solidaria a la economía informal. Philippe Adair (1989) señala que "Los tres componentes de la economía informal son la economía subterránea, la economía doméstica y la economía solidaria. Esta última se caracteriza por producir bienes y servicios no monetarios que circulan en ámbitos de sociabilidad según los principios de reciprocidad y redistribución" (1989, p. 3).

La economía solidaria es un término nuevo, elaborado con identidad propia, que da cuenta de uno de los mayores cambios ocurridos en los últimos años en todo el mundo, esto es: la irrupción de experiencias económicas solidarias guiadas por una racionalidad alternativa en el contexto de una fuerte crisis de legitimidad del modelo de desarrollo imperante.

\section{La economía solidaria en Colombia}

La formalización del modelo solidario en Colombia es reciente, pues se inició en 1931 con la expedición de la ley 134. Más adelante, en 1986, se adopta el concepto de economía solidaria con el Decreto 2536 del 4 de agosto, que dio vida al Consejo Nacional de Economía Solidaria y lo reconoce como un sector de gran importancia en el entorno económico nacional. 
Finalmente, en 1988, con la ley 79, se organizan las formas solidarias del cooperativismo, asociaciones mutuales y fondos de empleados (Superintendencia de la Economía Solidaria, 2007).

Hasta 1999, la función de supervisión de las entidades de economía solidaria fue adelantada por el Departamento Administrativo Nacional de Cooperativas (DANCOOP), la institución de gobierno encargada de definir la política para las formas solidarias de organización, en particular para el sector cooperativo. Pero con la expedición de la ley 454 de 1998 se transformó al DANCOOP en el Departamento Administrativo de la Economía Solidaria (DANSOCIAL), y se creó la Superintendencia de la Economía Solidaria (su-
PERSOlidaria) y el Fondo de Garantías del Sector Cooperativo (FOGACOOP).

En la actualidad, se reportan 10.055 entidades del sector solidario, que asocia a 5.453 .930 personas. Este sector viene de un período de crecimiento que le permite ser sostenible en tiempos de crisis, si se actúa en forma correcta, ya que la crisis económica mundial muestra sus primeros efectos en Colombia, y el sector solidario no es ajeno a ellos.

Existe un grupo de organismos de carácter público que planifica y desarrolla los programas de crecimiento del sector y sus entidades de control y vigilancia. Estas entidades se resumen en el siguiente cuadro (ley 454 de 1998. Artículos: 20 a 30,33 a 36, y 51).

Cuadro 1. Entidades de control y vigilancia del sector solidario en Colombia.

\begin{tabular}{|c|c|}
\hline \multicolumn{2}{|r|}{$\begin{array}{l}\text { Superintendencia de Economía Solidaria. Creada por la ley } 454 \text { de 1998, con los objetivos principales } \\
\text { de ejercer control, inspección y vigilancia sobre las entidades que cobijan su acción para asegurar el cum- } \\
\text { plimiento de las disposiciones legales y reglamentarias y de las normas contenidas en sus propios estatutos; } \\
\text { vigilar la correcta aplicación de los recursos de estas entidades, supervisar el cumplimiento del propósito } \\
\text { socioeconómico no lucrativo de las entidades vigiladas y demás funciones que la ley determine. }\end{array}$} \\
\hline 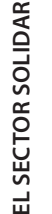 & $\begin{array}{l}\text { Departamento Administrativo Nacional de la Economía Solidaria. En 1998, con la ley } 454 \text { de 1998, } \\
\text { el Departamento Administrativo Nacional de Cooperativas fue transformado en el Departamento Admi- } \\
\text { nistrativo Nacional de la Economía Solidaria (DANSocIAL). Su objetivo es dirigir y coordinar la política esta- } \\
\text { tal para la promoción, planeación, protección, fortalecimiento y desarrollo empresarial de las organizacio- } \\
\text { nes de la economía solidaria y dar cumplimiento a las disposiciones establecidas en la Constitución Políti- } \\
\text { ca de Colombia. }\end{array}$ \\
\hline 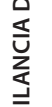 & $\begin{array}{l}\text { Consejo Nacional de Economía Solidaria. El Consejo Nacional de Economía Solidaria (cones) fue rees- } \\
\text { tructurado en } 1998 \text { con la ley 454. Este organismo formula y coordina a nivel nacional las políticas, estrate- } \\
\text { gias, planes, programas y proyectos generales pertinentes al sistema de economía solidaria. }\end{array}$ \\
\hline 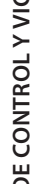 & $\begin{array}{l}\text { Fondo de Fomento de la Economía Solidaria. El Fondo de Fomento de la Economía Solidaria (FonES) se } \\
\text { creó con la ley } 454 \text { de 1998, vinculado a DANSocial y sometido a vigilancia de la Superintendencia de Econo- } \\
\text { mía Solidaria. Su función es la de otorgar créditos para los proyectos de desarrollo de las entidades de eco- } \\
\text { nomía solidaria, administrando los recursos que tiene a su disposición con los aportes de las entidades afi- } \\
\text { liadas. }\end{array}$ \\
\hline 㟧 & $\begin{array}{l}\text { Fondo de Garantías para las Cooperativas Financieras y de Ahorro y Crédito: Es un organismo de } \\
\text { carácter financiero vinculado al Ministerio de Hacienda y Crédito Público que está a cargo de mantener la } \\
\text { confianza de los ahorradores de las entidades cooperativas financieras inscritas, con el fin de salvaguardar } \\
\text { la estabilidad financiera y económica del sector. }\end{array}$ \\
\hline
\end{tabular}

Fuente: ley 454 de 1998. Artículos 20 a 30, 33 a 36, y 51. 
Otras formas asociativas de la economía solidaria, como son las empresas comunitarias, los fondos de empleados y las asociaciones mutualistas, han tomado como parte de su normatividad a las doctrinas, tradiciones y tecnologías cooperativas, adaptándolas a sus métodos y prácticas empresariales y organizacionales.

El hecho de ser el sector Cooperativo predominante dentro de la economía solidaria le permite ejercer un indiscutible liderazgo favorable en un todo para dinamizar su desarrollo y para mejorar su capacidad estratégica dentro de la economía nacional.

\section{Comportamiento económico del sector cooperativo en Colombia}

El cooperativismo en Colombia representa un importante agente para el desarrollo, ya que en la actualidad está involucrado en diversas actividades económicas. Además, desde el año 2000, el cooperativismo colombiano ha experimentado un crecimiento en número de entidades y en su base de asociados.

En la actualidad se reportan 7.833 empresas cooperativas que asocian a 4.473 .514 y emplean alrededor de 123.643 a tiempo completo. Tal como se muestra en la siguiente tabla.

Tabla 1. Evolución de las principales variables del sector cooperativo colombiano.

\begin{tabular}{|c|c|c|c|c|c|c|c|c|}
\hline \multicolumn{7}{|c|}{$\begin{array}{c}\text { CONFEDERACIÓN DE COOPERATIVAS DE COLOMBIA } \\
\text { EVOLUCIÓN DE LAS PRINCIPALES VARIABLES }\end{array}$} \\
\hline Año & $\begin{array}{c}\text { No. } \\
\text { Entidades }\end{array}$ & Activos & Pasivos & Patrimonio & Ingresos & Excedentes & Asociados & Empleados \\
\hline 2000 & 3.472 & 4.772 .886 & 2.466 .781 & 2.306 .105 & 5.790 .995 & 180.401 & 2.439 .122 & 51.085 \\
\hline 2001 & 3.517 & 6.034 .635 & 3.476 .196 & 2.558 .440 & 6.628 .317 & 220.866 & 1.617 .898 & 55.530 \\
\hline 2002 & 4.195 & 8.174 .549 & 4.868 .028 & 3.306 .521 & 9.357 .078 & 264.639 & 2.430 .649 & 69.030 \\
\hline 2003 & 5.107 & 9.492 .518 & 5.650 .645 & 3.841 .873 & 11.557 .137 & 305.381 & 2.602 .146 & 80.987 \\
\hline 2004 & 5.931 & 10.755 .077 & 6.326 .423 & 4.431 .450 & 13.198 .504 & 292.786 & 3.024 .101 & 93.945 \\
\hline 2005 & 6.462 & 12.467 .475 & 7.406 .841 & 5.066 .830 & 14.945 .484 & 294.540 & 3.305 .433 & 107.986 \\
\hline 2006 & 6.877 & 13.857 .713 & 8.080 .137 & 5.777 .576 & 17.205 .682 & 349.919 & 3.682 .496 & 114.924 \\
\hline 2007 & 7.349 & 16.871 .647 & 10.255 .902 & 6.615 .896 & 20.230 .356 & 373.914 & 4.020 .334 & 111.951 \\
\hline 2008 & 7.833 & 19.372 .178 & 11.722 .624 & 7.649 .554 & 22.583 .734 & 393.217 & 4.473 .514 & 123.643 \\
\hline
\end{tabular}

Cifras monetarias en millones de pesos

Fuentes: SIAC Confecoop, Superfinanciera, Supervigilancia, Superservicios, Saludcoop, Acemi, Gestar Salud.

En el ranking de las mil empresas más grandes de Colombia realizado por la revista Semana en su edición núm. 1.409, del 4 de mayo de 2009, que usa como variable para hacer el escalafón el monto de las ventas anuales de 2008, se encuentran cinco entidades del sector cooperativo.

La primera de ellas es el grupo SALUDCOOP, ocupando el puesto 18, con ventas por $\$ 2,97$ billones; la segunda es 
COOMEVA EPs, entidad de propiedad cooperativa, con ventas por $\$ 1,52$ billones, en el puesto 30; la tercera es COLANTA, con ventas por $\$ 1,38$ billones, en el puesto 35; la cuarta es COPIDROGAs, con ventas por $\$ 846$ mil millones, en el puesto 77 ; y la quinta es COPSERVIR, con ventas por $\$ 695$ mil millones, en el puesto 75 .

Igualmente, la publicación hace un escalafón especial de las veinticinco mayores empleadoras del país, encontrándose SALUDCOOP en primer lugar con 27.889 empleados; en el puesto 14, COOMEVA EPS, con 5.337; en el 18, COPSERVIR, con 4.849; y en el puesto 19, Colanta, con 4.675. La publicación destaca que SALUDCOOP no sólo es el grupo empresarial que más trabajadores emplea, sino el que generó el mayor número de puestos de trabajo en 2008, con 2.102.

También hace un escalafón de las cincuenta entidades financieras más grandes del país por nivel de ingresos de intereses, apareciendo en el listado Financiera COOMEVA puesto 19; J.F. KENNEDY, puesto 29; CONFIAR, puesto 33; COTRAFA, puesto 40; Cooperativa Financiera de Antioquia, puesto 43; COOFINEP, puesto 46; y JURISCoop Financiera, puesto 50.

Además, la revista publica para las entidades financieras a los primeros veinte puestos en diversos indicadores, y allí nuevamente se destacan algunas cooperativas. Por ejemplo, en materia de rentabilidad sobre el patrimonio, la cooperativa financiera J.F. KENNEDY ocupa el puesto 12; en calidad de cartera aparecen la cooperativas C.F.A en el puesto 6 , JURISCOOP Financiera en el puesto 8, CONFIAR en el puesto 13 y J.F KENNEDY en el puesto 17.

En el ramo de seguros generales se hace un escalafón con el monto de primas emitidas durante el 2008, apareciendo la ASEGURADORA SOLIDARIA DE COLOMBIA en el puesto 11 , con $\$ 212.344$ millones y LA EQUIDAD SEGUROS en el puesto 17, con $\$ 81.950$ millones, dentro de un universo de 24 compañías. Por su parte, en el ramo de seguros de vida LA EQUIDAD SEGUROs aparece en el puesto 13, con primas por $\$ 70.972$ millones, dentro de un total de diecinueve compañías.

Una de las secciones de la publicación está dedicada a modelos cooperativos exitosos, otorgando este reconocimiento a SALUDCOOP, COOMEVA, COLANTA Y LA EQUIDAD SEGUROS.

Para el año 2005 se cuenta con más de 9.400 entidades cooperativas, con ingresos de $\$ 16.009 .571$ millones, equivalentes a 5,61\% del рів. Considerando estas cifras, se demuestra la relevancia que tiene este sector en la economía colombiana. En la siguiente gráfica se evidencia la evolución de la participación de los ingresos del sector solidario en el PIB desde 1996 hasta el año gravable 2005. 
Gráfica 1. Participación de los ingresos del sector cooperativo en el PIB.

Años gravables 1996-2005.

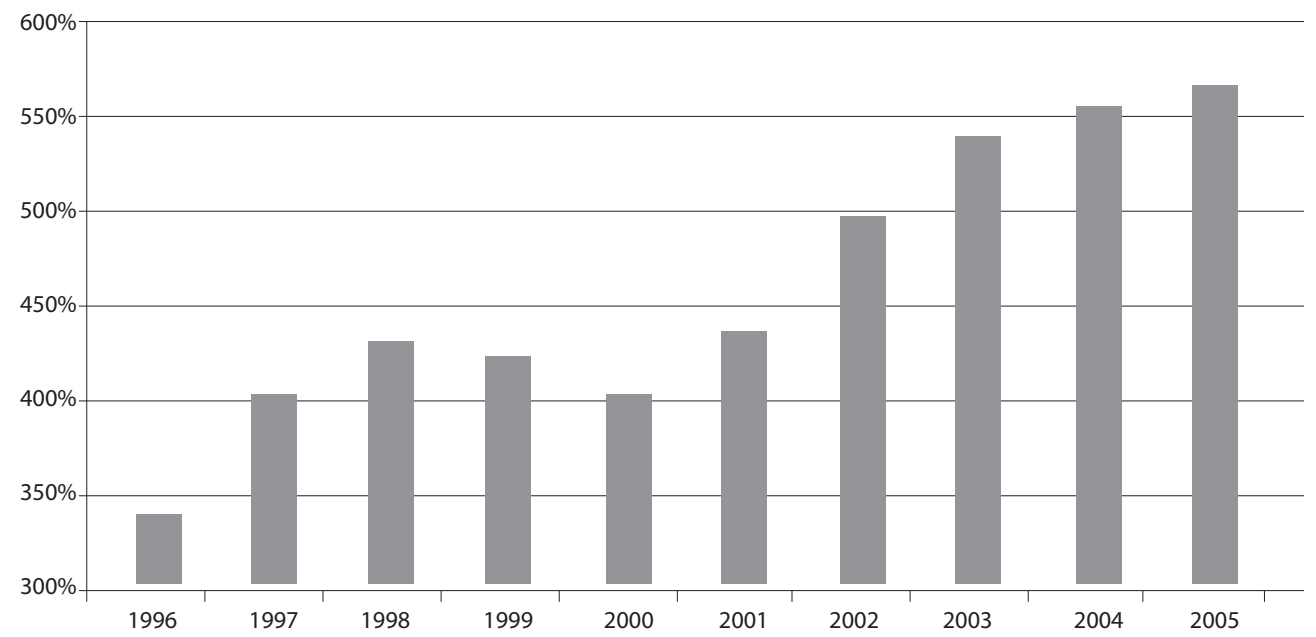

Fuente: Bodega de Datos. Declaraciones de Renta. Con corte a 2 de marzo de 2007. Oficina de Estudios Económicos. Supuestos Macroeconómicos de enero 25 de 2007. Viceministerio Técnico del Ministerio de Hacienda y Crédito Público. Elaboró: Oficina de Estudios Económicos. Dirección de Impuestos y Aduanas Nacionales.

La gráfica muestra que las cooperativas han mantenido un crecimiento sostenido marcado por la crisis económica que vivió el país a final de los años noventa.

La composición de los ingresos de las entidades cooperativas en el año 2005 es la variable relevante para catalogar los subsectores en orden de presentación. Según esta variable, los subsectores de comercio al por mayor, otros servicios, manufactura de alimentos, servicios financieros y comercio al por menor concentran el $88,90 \%$ de ingresos de las cooperativas. Es de recalcar que durante los once años en observación estos cinco subsectores han mantenido los primeros lugares en cuanto al nivel de ingresos del total de cooperativas.
Los subsectores de servicios de transporte, almacenamiento y comunicaciones; agropecuario, silvicultura y pesca; actividades deportivas; construcción; comercio al por mayor y al por menor de vehículos automotores; fabricación de sustancias químicas; manufactura de textiles; minero; fabricación de productos minerales e industria de la madera; servicios de hoteles y restaurantes; y el subsector de electricidad, gas y vapor constituyen el restante $11,10 \%$ de los ingresos percibidos por el total de entidades cooperativas.

En la siguiente gráfica se puede apreciar la composición porcentual de los ingresos de las cooperativas por subsector económico. 
Gráfica 2. Composición porcentual de los ingresos de las cooperativas por subsector económico.

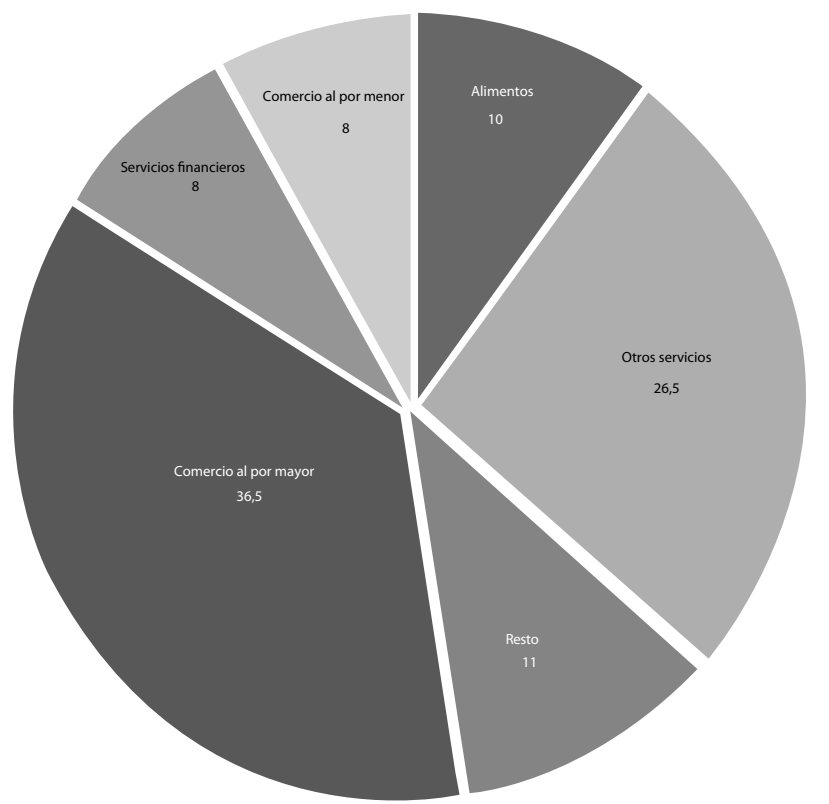

Fuente: Bodega de Datos. Declaraciones de Renta. Con corte a 2 de marzo de 2007. Oficina de Estudios Económicos. Elaboró: Oficina de Estudios Económicos. Dirección de Impuestos y Aduanas Nacionales.

Estos datos evidencian la capacidad que tiene el sector cooperativo de impactar de manera positiva en la actividad económica nacional y el papel que juega en la generación de empleo y en la propagación de programas de educación a sus asociados y a terceros. Los excelentes resultados que arrojan las cifras del sector cooperativo en los últimos años no sólo se deben atribuir a la recuperación de la confianza en los mercados financieros, o a la política de bajas de tasas de interés del Banco de la República, sino también a la fidelidad de los asociados como gestores, la estabilidad de los nichos de mercado de las cooperativas y el fortalecimiento patrimonial. Esto a través de la generación de excedentes cooperativos, que en ningún caso se reparten en beneficio particular de la propia base social, sino que se destinan al programa de educación del Gobierno. 


\section{Procesos de certificación de calidad para el sector cooperativo}

En el año 2005 se comenzó a ejecutar un convenio entre DANSOCIAL e ICONTEC, con el fin de construir documentos normativos que ayudaran como referencia para mejorar el proceso de calidad de las organizaciones del sector solidario. De allí surge la necesidad de realizar un esquema de certificación para que el sector solidario tenga la oportunidad de realizar la implementación de un sistema y pueda demostrar la conformidad con el mismo.

A raíz del incremento de las exigencias del mercado, en las organizaciones del sector solidario se hace cada día más necesario contar con estándares de calidad dentro de estas organizaciones. El sistema de gestión de la calidad permite, entre otros:

- Desarrollar una imagen que incremente la confianza entre las partes interesadas (empleados, asociados, afiliados, beneficiarios, voluntarios y miembros, entre otros) y los grupos de interés.

- Contribuir a la legitimación social del sector solidario en el país.

- Permitir el establecimiento de alianzas entre las organizaciones del sector solidario y actores del entorno que fortalezcan su desarrollo y sostenibilidad.

- Facilitar el fortalecimiento de las organizaciones del sector solidario como constructoras de valores sociales y como agentes de cambio económico, social, político y cultural.

- Lograr organizaciones solidarias más competitivas, que respondan a las necesidad de sus partes interesadas (empleados, asociados, afiliados, beneficiarios, voluntarios y miembros, entre otros) y grupos de interés.

- Proporcionar herramientas y soportes que orienten procesos de gestión en el sector solidario.

En la siguiente tabla se presentan las normas técnicas colombianas para la certificación de empresas del sector solidario. 
Tabla 2. Normas técnicas colombianas para la certificación de empresas del sector solidario.

\begin{tabular}{|c|c|c|}
\hline NORMA & TÍTULO & DESCRIPCIÓN \\
\hline NTC 5499 & $\begin{array}{l}\text { Sistema de gestión de } \\
\text { la democracia partici- } \\
\text { pante en las organiza- } \\
\text { ciones solidarias. }\end{array}$ & $\begin{array}{l}\text { Busca, principalmente, identificar y fortalecer los espacios organizacionales de } \\
\text { participación y buenas prácticas en la toma de decisiones, orientando a las or- } \\
\text { ganizaciones solidarias al cumplimiento de sus principios. } \\
\text { En ella se establecen los requisitos para la implementación, operación y mante- } \\
\text { nimiento de un sistema de gestión de la democracia participante para las orga- } \\
\text { nizaciones del sector solidario. } \\
\text { Para el cumplimiento de esta norma, la organización debe establecer, docu- } \\
\text { mentar y mantener un sistema de gestión para la democracia; suministrar los } \\
\text { medios para mejorar continuamente su eficacia; elaborar y documentar el có- } \\
\text { digo para la democracia, basado en la misión, visión, principios y valores pro- } \\
\text { pios de la entidad como un conjunto de políticas que incorporen los principios } \\
\text { y requisitos establecidos por esta norma; establecer los procesos (fases, activi- } \\
\text { dades, responsables, procedimientos y métodos de seguimiento y control, en- } \\
\text { tre otros) necesarios para lograr la participación de las partes interesadas en la } \\
\text { organización; propiciar los procesos necesarios y adecuados para que las partes } \\
\text { interesadas participen en el diseño de su estrategia y contribuyan a la construc- } \\
\text { ción de su planificación y desarrollo; propiciar el conocimiento y la divulgación } \\
\text { de la visión, misión, los principios y los valores, el código para la democracia, su } \\
\text { adhesión a los mismos y el sentido de pertenencia; garantizar la participación y } \\
\text { la transparencia en las asambleas y demás órganos de gobierno y gestión; do- } \\
\text { cumentar los procesos y procedimientos de funcionamiento del sistema para el } \\
\text { ejercicio de la democracia y definir los informes asociados; y cumplir con la re- } \\
\text { glamentación legal y estatutaria vigente sobre el tema de elecciones, participa- } \\
\text { ción y toma de decisiones, de acuerdo con el tipo de organización. }\end{array}$ \\
\hline GTC 192 & $\begin{array}{l}\text { Modelo de sistema de } \\
\text { gestión para coope- } \\
\text { rativas. }\end{array}$ & $\begin{array}{l}\text { Este modelo suministra directrices para establecer un modelo de gestión cuyo } \\
\text { fin es planear, hacer, verificar y mejorar los procesos y los resultados de las coo- } \\
\text { perativas, independientemente del tamaño y del sector o actividad que desa- } \\
\text { rrolle. Se basa en algunos lineamentos para la constitución, funcionamiento y } \\
\text { desarrollo de las cooperativas: tener en cuenta que los procesos cumplan el ci- } \\
\text { clo planear, hacer, verificar y actuar; seguir la secuencia de las actividades de la } \\
\text { cooperativa; considerar los elementos comunes en las diferentes organizacio- } \\
\text { nes; y definir las características de los procesos, las actividades y su enfoque, te- } \\
\text { niendo como referencia la reglamentación pertinente. }\end{array}$ \\
\hline GTC 193 & $\begin{array}{l}\text { Modelo de gestión } \\
\text { para organizaciones de } \\
\text { acción voluntaria. }\end{array}$ & $\begin{array}{l}\text { Esta guía suministra orientación a las organizaciones de voluntariado (ODV), en- } \\
\text { tidades con acción voluntaria (ECAV) y colectivos que estén realizando o están } \\
\text { interesados en realizar acciones voluntarias para el establecimiento de un mo- } \\
\text { delo que permita dirigir y administrar sus procesos y sus resultados. Esta guía } \\
\text { consta de directrices y recomendaciones, y no tiene la intención de ser utiliza- } \\
\text { da para propósitos reglamentarios, ni contractuales, ni de certificación; es de } \\
\text { carácter voluntario y su aplicación no exime a las organizaciones de acción vo- } \\
\text { luntaria del cumplimiento de las disposiciones legales vigentes aplicables, y no } \\
\text { es contraria a las mismas. }\end{array}$ \\
\hline GTC 194 & $\begin{array}{l}\text { Guía para la consolida- } \\
\text { ción de la memoria ins- } \\
\text { titucional en organiza- } \\
\text { ciones del sector soli- } \\
\text { dario. }\end{array}$ & $\begin{array}{l}\text { Esta guía técnica colombiana establece directrices para recopilación, recupe- } \\
\text { ración, organización, conservación y preservación de la memoria institucional } \\
\text { en las organizaciones del sector solidario, con el fin de facilitar el acceso a la in- } \\
\text { formación por parte de la organización, grupos de interés y partes interesadas. }\end{array}$ \\
\hline
\end{tabular}




\begin{tabular}{|c|c|c|}
\hline NORMA & TíTULO & DESCRIPCIÓN \\
\hline GTC 195 & $\begin{array}{l}\text { Sistema de informa- } \\
\text { ción, comunicación y } \\
\text { divulgación en las or- } \\
\text { ganizaciones del sector } \\
\text { solidario. }\end{array}$ & $\begin{array}{l}\text { Esta guía establece directrices para la implementación y la operación de un sis- } \\
\text { tema de información, comunicación y divulgación en las organizaciones del } \\
\text { sector solidario. }\end{array}$ \\
\hline GTC 196 & $\begin{array}{l}\text { Rendición de cuentas } \\
\text { en organizaciones del } \\
\text { sector solidario. }\end{array}$ & $\begin{array}{l}\text { Esta norma técnica para la rendición de cuentas en las organizaciones del sec- } \\
\text { tor solidario es una herramienta para generar espacios de encuentro entre la or- } \\
\text { ganización solidaria, las partes interesadas y los grupos de interés, con el fin de } \\
\text { implementar mecanismos de información y retroalimentación sobre la opera- } \\
\text { ción de las organizaciones solidarias. } \\
\text { Para la implementación de la rendición de cuentas es indispensable que la or- } \\
\text { ganización cuente con un sistema de información, comunicación y divulgación } \\
\text { de acuerdo con lo establecido en la GTc 195-2007: Sistema de información, co- } \\
\text { municación y divulgación en las organizaciones del sector solidario. }\end{array}$ \\
\hline GTC 197 & $\begin{array}{l}\text { Directrices para la ca- } \\
\text { pacitación, formación, } \\
\text { promoción, asistencia } \\
\text { técnica e investigación } \\
\text { de la base social. }\end{array}$ & $\begin{array}{l}\text { Esta guía busca brindar herramientas para formular y operar la educación soli- } \\
\text { daria como factor de cambio para el desarrollo sostenible del sector solidario a } \\
\text { través del diagnóstico, diseño, evaluación, implementación y mejoramiento del } \\
\text { Proyecto Educativo Socioempresarial (PESEM). } \\
\text { Un proceso planificado y sistemático de educación solidaria puede contribuir } \\
\text { de manera importante a una organización para fortalecer su base social, mejo- } \\
\text { rar sus capacidades y lograr sus objetivos organizacionales. }\end{array}$ \\
\hline GTC 198 & $\begin{array}{l}\text { Sistema de gestión de } \\
\text { la calidad para entida- } \\
\text { des acreditadas por } \\
\text { DANSOCIAL para impartir } \\
\text { educación solidaria re- } \\
\text { comendaciones }\end{array}$ & $\begin{array}{l}\text { Este documento normativo establece las directrices de un sistema de gestión } \\
\text { de la calidad para ser implementado en entidades acreditadas por DANSOCIAL que } \\
\text { brindan servicios de educación solidaria. Para la aplicación de este documento } \\
\text { normativo es indispensable tener en cuenta la norma NTC-Iso 9000:2005: Siste- } \\
\text { mas de Gestión de la Calidad: Fundamentos y Vocabulario. }\end{array}$ \\
\hline DE $296 / 06$ & $\begin{array}{l}\text { Guía para la interpre- } \\
\text { tación de sistemas de } \\
\text { gestión de la calidad en } \\
\text { organizaciones del sec- } \\
\text { tor solidario. }\end{array}$ & $\begin{array}{l}\text { Este documento establece directrices para implementar un sistema de gestión } \\
\text { de la calidad en organizaciones del sector solidario cuyo fin es contribuir al for- } \\
\text { talecimiento y mejoramiento de la calidad en los procesos y resultados de es- } \\
\text { tas organizaciones. } \\
\text { No establece ningún requisito, ni amplía ni modifica de ninguna forma los re- } \\
\text { quisitos de la norma iso 9001:2000; sólo busca servir de ayuda para su inequí- } \\
\text { voca interpretación y orientación de las personas que desarrollan la gestión en } \\
\text { las organizaciones del sector solidario. Se han incluido cada uno de los nume- } \\
\text { rales de la norma iso } 9001: 2000 \text { y se recomienda que la guía de interpretación se } \\
\text { lea en conjunto con el numeral de iso } 9001: 2000 \text {, puesto que en algunos casos } \\
\text { donde el texto del numeral es lo suficientemente claro y explícito sólo se ofre- } \\
\text { ce una breve interpretación. }\end{array}$ \\
\hline
\end{tabular}

Fuente: www.icontec.org/Catalogo.asp.

\section{Diseño metodológico}

Para este documento se realizó un procedimiento basado en una investigación exploratoria descriptiva, enfocándolo a las empresas del sector cooperativo per-
Medellín. Este tipo de investigación es el más apropiado, ya que no existen estudios previos que sirvan como soporte para el inicio de un estudio estadístico de tipo cuantitativo. 
Para realizar el estudio se atendió la siguiente metodología. Con base en el listado de cooperativas certificadas entre los años 2005 y 2009 en Colombia, suministrado por el ICONTEC, se extrajeron las ciudades más representativas dentro del listado, en este caso Medellín con veinticinco entidades y Bogotá con dieciséis, para una participación del 26,00\% y del $17,00 \%$ respectivamente, lo que permitió focalizar el estudio objeto de este trabajo.

Se realizó seguidamente un modelo de encuesta, que se encuentra disponible en el link http://www.desarrolloweb.org/encuesta/index.php, y por medio de correo electrónico se envió a las entidades seleccionadas para el estudio. Adicionalmente, fueron realizadas entrevistas vía telefónica y visitas a cooperativas situadas en Bogotá.

Con base en el punto anterior, se determinó un método de selección relativamente aleatorio, pues pese a que todas las empresas tenían la misma opción de ser elegidas, el estudio se limitó sólo a aquellas cooperativas que colaboraron con el diligenciamiento de las encuestas.

Se recibieron respuestas de veintidós entidades. $61,00 \%$ de los resultados correspondieron a empresas situadas en la ciudad de Medellín (trece empresas) y el $39,00 \%$ en la ciudad de Bogotá (nueve empresas).

Posteriormente, se tabuló la información de las encuestas para realizar un estudio cualitativo que buscaba medir el comportamiento de variables relativas al tipo de empresa certificada, tiempo que lleva certificada, motivación para la certificación e incidencia de la certificación en sus procesos. Los resultados de estas encuestas se analizaron para la construcción del presente documento, con el que se pretende servir como herramienta para otras cooperativas que deseen ser certificadas.
Después de la unificación de la información y el análisis utilizando herramientas propias de la estadística descriptiva, se obtuvieron las conclusiones al caso.

\section{Discusión de resultados}

\section{Identificación de la cooperativa}

\section{Tipo de cooperativas según el desarrollo de sus actividades}

El 75\% de las cooperativas que respondieron la encuesta son especializadas, el $20 \%$ son cooperativas multiactivas y el $5 \%$ son cooperativas integrales.

Gráfica 3. Tipo de cooperativa.

2-3. Seleccione el tipo de Cooperativa según el desarrollo de sus actividades

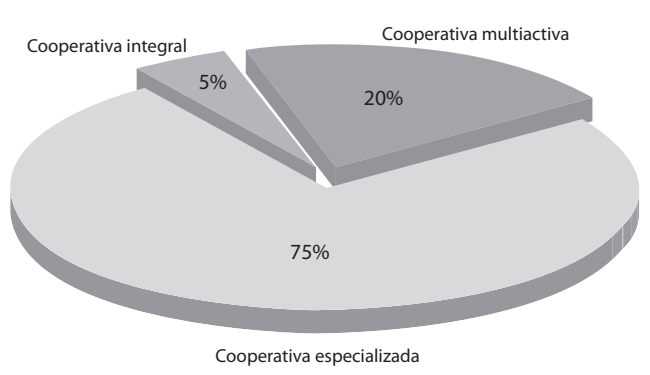

Fuente: la investigación.

\section{Tipo de cooperativas según}

\section{su actividad económica}

El 38,1\% de las cooperativas que contestaron la encuesta fueron cooperativas de trabajo asociado; el 23,81\% son de ahorro y crédito; el 14, 29\%, de salud; el $9,52 \%$, de transporte; el 4,76\%, agrícolas; el 4,76\%, de servicios públicos; y el $4,76 \%$, de desarrollo comunitario. 
Gráfica 4. Cooperativa según su actividad económica.

2-3. Seleccione el tipo de Cooperativa según el desarrollo actividad económica

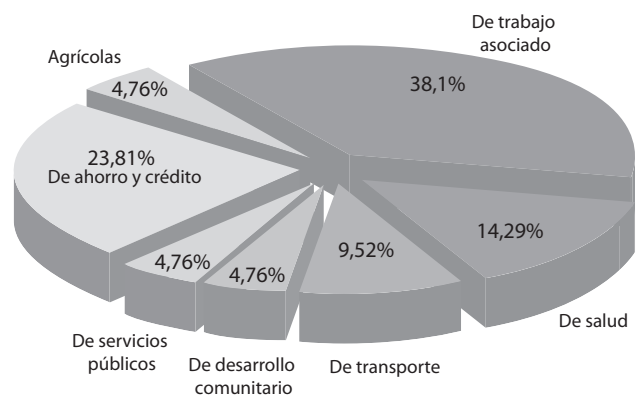

\section{Acerca del proceso de certificación de la cooperativa}

\author{
Año en que la cooperativa \\ recibió la más reciente \\ certificación de ICONTEC
}

El 11,1\% la recibieron en 2005; el $27,78 \%$, en 2006 ; el $44,4 \%$, en 2007 ; el $11,1 \%$, en 2008 ; y $5,56 \%$, en 2009 .

\section{Tipo de certificación ICONTEC que obtuvo la cooperativa}

El 85,71\% obtuvo la certificación de sistemas de gestión, y el 14,29\%, la de certificación del producto.

De acuerdo con un sistema de evaluación de calificación, siendo 1 mínimo y 5 máximo, se obtuvieron las siguientes motivaciones:

Tabla 3. Calificación de las motivaciones de la cooperativa para obtener una certificación.

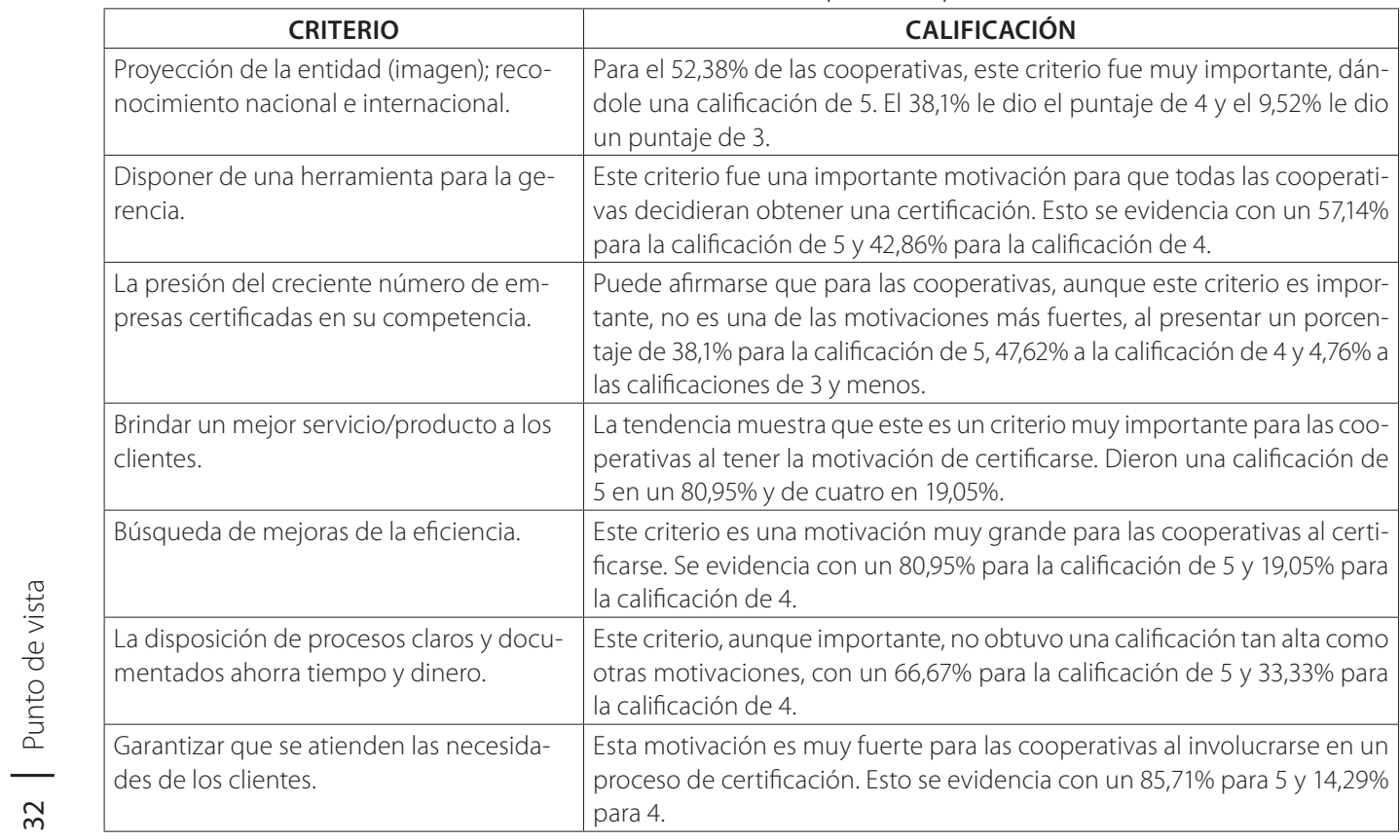


Al promediar los puntajes obtenidos en esta pregunta, obtenemos que las principales motivaciones de las cooperativas para obtener una certificación de calidad son aquellas relacionadas con la satisfacción y el brindar mejores productos y servicios a los clientes. Los promedios de estas clasificaciones se presentan a continuación.

Tabla 4. Promedio de las calificaciones de las motivaciones de las cooperativas para obtener una certificación.

\begin{tabular}{|l|c|}
\hline \multicolumn{1}{|c|}{ MOTIVACIÓN } & CALIFICACIÓN \\
\hline Garantizar que se atienden las necesidades de los clientes. & 4,85 \\
\hline Brindar un mejor servicio/producto a los clientes. & 4,8 \\
\hline Búsqueda de mejoras de la eficiencia. & 4,8 \\
\hline La disposición de procesos claros y documentados ahorra tiempo y dinero. & 4,6 \\
\hline Disponer de una herramienta para la gerencia. & 4,57 \\
\hline Proyección de la entidad (imagen); reconocimiento nacional e internacional. & 4,42 \\
\hline La presión del creciente número de empresas certificadas en su competencia. & 4,09 \\
\hline
\end{tabular}

Tabla 5. Calificación del nivel de importancia (1 para la mínima, 5 para la máxima) de los principios de gestión de la calidad que, consideran las cooperativas, se tuvieron en cuenta en el proceso para la certificación.

\begin{tabular}{|c|c|}
\hline CRITERIO & CALIFICACIÓN \\
\hline Enfoque al cliente. & $\begin{array}{l}\text { Este principio fue uno de los más importantes para las cooperativas, ya } \\
\text { que obtuvo } 85,71 \% \text { para la calificación de } 5 \text { y } 14,29 \% \text { para la de } 4 \text {. }\end{array}$ \\
\hline Liderazgo. & $\begin{array}{l}\text { Este principio es importante, y sus calificaciones fueron } 61,9 \% \text { para la cali- } \\
\text { ficación de } 5 \text { y } 38,1 \% \text { para la calificación de } 4 \text {. }\end{array}$ \\
\hline Participación del personal. & $\begin{array}{l}\text { Este principio también es considerado importante para las cooperativas, } \\
\text { ya que todas sus calificaciones estuvieron en los puntajes } 4 \text { y 5, con un } \\
52,38 \% \text { para la calificación de } 5 \text { y 47,62\% para la calificación de } 4 \text {. }\end{array}$ \\
\hline Enfoque basado en procesos. & $\begin{array}{l}\text { Esta motivación no obtuvo calificaciones tan altas, pero sigue siendo con- } \\
\text { siderada importante; con un porcentaje de } 57,14 \% \text { para la calificación de } 5 \text {, } \\
38,1 \% \text { para la calificación de } 4 \text { y 4,76\% para la calificación de } 3 \text {. }\end{array}$ \\
\hline Enfoque de sistema para la gestión. & $\begin{array}{l}\text { Esta motivación, igual que la anterior, no obtuvo calificaciones tan altas, } \\
\text { pero sigue siendo importante; con un porcentaje de } 61,9 \% \text { para la califica- } \\
\text { ción de 5, 33,3\% para la calificación de } 4 \text { y 4,76\% para la calificación de } 3 \text {. }\end{array}$ \\
\hline Mejora continua. & $\begin{array}{l}\text { Esta motivación obtuvo un porcentaje de 61,9\% para la calificación de } 5 \text { y } \\
38,1 \% \text { para la calificación de } 4 \text {. }\end{array}$ \\
\hline $\begin{array}{l}\text { Enfoque basado en hechos para la toma } \\
\text { de decisiones. }\end{array}$ & $\begin{array}{l}\text { Esta motivación tuvo un porcentaje muy alto para la calificación de 5, con } \\
\text { un } 71,43 \%, 23,81 \% \text { para la calificación de } 4 \text { y 4,76\% para la calificación de } 3 \text {. }\end{array}$ \\
\hline $\begin{array}{l}\text { Relaciones mutuamente beneficiosas con } \\
\text { el proveedor. }\end{array}$ & $\begin{array}{l}\text { Esta motivación no presentó calificaciones tan altas, con un 57,14\% para la } \\
\text { calificación de 5, 33,3\% para la calificación de 4, 4,76\% para la calificación } \\
\text { de } 3 \text { y 4,76\% para la calificación de } 2 \text {. }\end{array}$ \\
\hline
\end{tabular}

Al promediar los puntajes obtenidos en esta pregunta, obtenemos que el principio de gestión de calidad más tenido en cuenta por las cooperativas fue el enfoque en el cliente. 
Con respecto a las normas técnicas que tuvo en cuenta la cooperativa para su proceso de certificación, la más revisada por las organizaciones fue la GTC 192: Modelo de sistema de gestión para las cooperativas, con un 41,2\%, seguida de la 296/06: Guía para la interpretación de sistemas de gestión de calidad en organizaciones del sector solidario. Un 11,8\% de las cooperativas tuvo en cuenta la norma GTC 198: Sistema de gestión de calidad para las entidades acreditadas por DANSOCIAL para impartir educación solidaria: Recomendaciones. Un 23.5\% marcaron la opción de otro, y en ella hacían referencia a la Norma Técnica Iso 9001 y 9004.

Al promediar los puntajes obtenidos en esta pregunta, obtenemos que los dos cambios principales que se perciben en las cooperativas después del proceso de certificación son las mejores prácticas en el trabajo y una mejor actitud ante las reclamaciones de los clientes.

Los promedios de estas clasificaciones se presentan a continuación.

Tabla 6. Promedio de las calificaciones de los cambios

ocasionados gracias al proceso de certificación.

\begin{tabular}{|l|c|}
\hline \multicolumn{1}{|c|}{ MOTIVACIÓN } & CALIFICACIÓN \\
\hline $\begin{array}{l}\text { La revisión de las prácticas y patrones tradicionales ocasionó mejores prácticas en el tra- } \\
\text { bajo. }\end{array}$ & 4,55 \\
\hline Mejor actitud ante las reclamaciones de los clientes. & 4,55 \\
\hline $\begin{array}{l}\text { El desarrollo de una visión de conjunto de la institución que supera a la visión aislada de } \\
\text { cada uno sobre su propia área de trabajo. }\end{array}$ & 4,45 \\
\hline Desarrolló la cultura de consulta a la documentación de los procesos. & 4,4 \\
\hline Una gestión del recurso humano más conectada con los objetivos organizacionales. & 4,4 \\
\hline Un liderazgo participativo, creativo e innovador. & 4,3 \\
\hline
\end{tabular}

Al promediar los puntajes obtenidos en esta pregunta, obtenemos que los dos beneficios principales que perciben las cooperativas después del proceso de certificación son el incremento en la calidad de los servicios y/o productos prestados y la planificación, organización y control de las acciones.

Los promedios de estas clasificaciones se presentan a continuación.

Tabla 7. Beneficios que perciben las cooperativas al obtener la certificación.

\begin{tabular}{|c|c|}
\hline MOTIVACIÓN & CALIFICACIÓN \\
\hline Incremento en la calidad de los servicios y/o productos prestados. & 4,66 \\
\hline Planificación, organización y control de las acciones. & 4,57 \\
\hline Satisfacción de los clientes. & 4,52 \\
\hline Objetivos y metas claramente definidos. & 4,47 \\
\hline Mejoría en la imagen institucional y su credibilidad. & 4,47 \\
\hline Incremento en el número de clientes. & 4,33 \\
\hline Oferta de servicios y/o productos adecuados a las necesidades reales de los clientes. & 4,33 \\
\hline Optimización de recursos. & 4,33 \\
\hline Mejor comunicación entre los departamentos. & 4,33 \\
\hline
\end{tabular}




\begin{tabular}{|l|c|}
\hline \multicolumn{1}{|c|}{ MOTIVACIÓN } & CALIFICACIÓN \\
\hline Menos conflictos internos y mayor involucramiento entre diferentes áreas. & 4,14 \\
\hline Reducción de costos. & 4,14 \\
\hline Mejoramiento del clima organizacional. & 4,09 \\
\hline
\end{tabular}

Además se señaló si consideraban que existía algún beneficio adicional que le haya dejado el obtener la certificación. El 80\% de las cooperativas encuestadas informaron no haber obtenido ningún beneficio adicional en el proceso de certificación. De los anteriormente nombrados, un $20 \%$ contestaron que sí recibieron beneficios adicionales, pero al preguntar cuáles fueron éstos, no especificaron ninguna información.

En cuanto a las dificultades presentadas por las cooperativas en el proceso de certificación, las cooperativas presentaron las siguientes:

- Es difícil el proceso de toma de conciencia por parte del personal, ya que existe resistencia a los cambios.

- En algunos casos se identificó que faltaba liderazgo de los líderes del proceso al interior de las cooperativas.

- Las cooperativas manifestaron que no hay claridad por parte del equipo auditor del ICONTEC entre las diferencias de las empresas privadas y las del sector cooperativo.

- Otras cooperativas manifestaron que mantener las condiciones de la certificación no es una tarea sencilla, pero que entre mayor sea el tiempo de certificados es más sencillo mantener los cambios, ya que sí se alcanza una cultura organizacional en este sentido.

Como información que se sugiere debe ser incluida en las normas técnicas de calidad, y que las cooperativas certificadas aportarían después del proceso de certificación, se presentaron los siguientes aportes:

- Se debe considerar fusionar la iso 9001 y la Iso 9004, para garantizar que realmente las empresas gestionen la mejora continua.

- Para poder realizar un mejor ejercicio de auditoría, es importante que los auditores conozcan acerca del sector solidario, acerca de las cooperativas y acerca de las cooperativas de trabajo asociado.

En cuanto a la satisfacción de las cooperativas con el proceso realizado para la certificación, en general, el porcentaje de satisfechas con el proceso de certificación es muy alto, ya que alcanza un 95\% frente a un 5\% de cooperativas no satisfechas. Las que plantean que sí están satisfechas informan que este proceso les ha traído beneficios, que la certificación ha sido un medio para alcanzar la calidad y la optimización de recursos. En tanto que las que plantean que no se encuentran satisfechas consideran que el proceso de certificación está demasiado enfocado a la eficacia.

\section{Conclusiones}

- Las empresas cooperativas certificadas en Bogotá y Medellín realizan, en su gran mayoría, actividades de 
servicios. Este tipo de empresas tiene como finalidad principal la satisfacción de las necesidades de los clientes y la búsqueda del beneficio en general de la sociedad, por lo cual la certificación en calidad se convierte en la herramienta más adecuada para lograrlo.

- En los últimos años ha incrementado el número de cooperativas que han logrado la certificación de sus procesos de calidad, siendo la certificación en sistemas de gestión la preferida por las empresas, ya que ésta es la que más se acopla a la motivación de las entidades por brindar un mejor servicio o producto a los clientes, mejorar la eficiencia en sus procesos y ganar mayor participación en el mercado.

- Para las empresas cooperativas, el mayor inconveniente en el proceso de certificación se relaciona con el proceso de auditoría, ya que los auditores no conocen al sector cooperativo y sus especificidades.

- Lastimosamente, los documentos normativos del sector solidario no son muy tenidos en cuenta por las cooperativas en su proceso de certificación, ya que éstas se enfocan directamente en la Norma de Sistema de Gestión de Calidad Iso. Sería muy importante que el ICONTEC diera a conocer más estas herramientas.

- Para las cooperativas, obtener una certificación en calidad es una práctica motivada especialmente en garantizar que se atiendan las necesidades de los clientes y brindarles un mejor servicio/producto. En este sentido, la investigación señala que el principio de gestión de calidad más tenido en cuenta por las cooperativas fue el enfoque en el cliente y la búsqueda de que el producto satisfaga sus necesidades, independientemente de que el producto sea el mejor del mercado.

- Las empresas cooperativas buscan certificarse en calidad porque es una herramienta que les indica que sus procesos y productos cumplen con los estándares básicos de calidad y, por lo tanto, están en condiciones de competir en el mercado, independientemente de la diferenciación del producto.

- La implementación de un sistema de calidad motivado en la satisfacción de los requerimientos de los clientes ha generado que las empresas cooperativas, a través de la mejora en sus prácticas empresariales y de sus procesos, evidencien el incremento en la calidad de los servicios y productos que ofrecen, por medio de la optimización de sus recursos y mejora en sus resultados.

- Los principales cambios que ocasiona el proceso de certificación en el ambiente y la cultura organizacional de las cooperativas son mejoras en las prácticas en el trabajo y una mejor actitud ante las reclamaciones de los clientes. Esto se debe a que el punto focal para la certificación es la satisfacción del cliente, incluso por encima de la diferenciación del producto.

- Gracias a la certificación, las empresas cooperativas encuestadas evidenciaron que se han convertido en compañías cuyas acciones se encuentran más planificadas y obedecen a objetivos previamente establecidos, por lo cual pueden realizar un mayor control de las mismas y medir sus resultados.

- La certificación en calidad, según los resultados del estudio realizado, es 
una herramienta que ayudó en el direccionamiento estratégico de las empresas, sirviendo como medio para esclarecer y organizar sus funciones, objetivos, metas, productos o servicios ofrecidos y tareas.

- La competitividad se encuentra asociada estrechamente con la capacidad que tienen las empresas para producir o vender sus bienes a costos más bajos que los de sus rivales. Sin embargo, el estudio demostró que, en la reducción de costos y la optimización de recursos, las empresas certificadas no obtuvieron beneficios significativos, por lo cual éste puede ser un factor que perjudique la competitividad de estas empresas en el mercado y, por lo tanto, se convierte en un punto focal a la hora de analizar y evaluar la eficiencia en los procesos de las empresas del sector cooperativo.

- Para tener mayor participación en el mercado, es necesario ofrecer productos que sean atractivos y adecuados a los deseos de los clientes; sin embargo, este es un aspecto en el que las cooperativas certificadas no tuvieron grandes beneficios. Esto obedece a que las empresas sólo buscan que sus productos cumplan estándares de calidad previamente establecidos, pero no le dan relevancia al valor agregado que debe tener para la diferenciación del mismo.

- Las cooperativas presentan dificultades en el proceso de certificación, principalmente en la toma de conciencia por parte del personal, ya que existe resistencia a los cambios y en la falta de liderazgo de los líderes del proceso al interior de las cooperativas. Por esto, es fundamental tener claro que, para implantar un sistema de calidad, todas las personas que hacen parte de la cooperativa deben estar sensibilizadas y formadas.

- El sector cooperativo es consciente de las ventajas que genera un proceso de certificación en su desarrollo organizacional. Sin embargo, este estudio permite concluir que la certificación no es una herramienta que garantice la competitividad, pero el cumplimiento de los requisitos normativos sí conduce a una mayor eficiencia y eficacia en los procesos, lo que con el tiempo genera ventajas competitivas con respecto a las demás empresas y cooperativas. Es por esto que alcanzar la certificación y la conformidad con la norma no deben ser considerados como un objetivo final en sí mismo; se debe considerar la utilización de la norma para consolidar su gestión.

- Los procesos de certificación son importantes para muchas empresas del sector cooperativo, sin embargo, es necesario sensibilizar, informar y motivar a quienes aún no son conscientes de los cambios del mercado en el que la calidad es la clave que permite la competitividad de todas las actividades económicas.

- A pesar de que con la implementación del sistema de calidad las empresas cooperativas de Bogotá y Medellín lograron cumplir su objetivo de satisfacer las necesidades de sus clientes, es importante esclarecer que esto no implica que sean más competitivas, ya que la competitividad está determinada por la diferenciación que tenga el producto ante los clientes, que los haga preferir el producto porque posee ciertas características que no tienen los de la competencia y que lo hacen mejor que el de sus rivales. En 
este sentido, la certificación en calidad se convierte en el medio, o en la ruta o el camino, que puede colaborar a llegar a ello.

- A fin de que la certificación en calidad se convierta en un instrumento para llegar a la competitividad, es necesario cambiar la visión que se tiene en su implementación. No hay que verla solamente bajo el punto de vista de satisfacción de necesidades al cliente, sino también hay que tener en cuenta la relevancia de aspectos como la mejora continua, optimización de recursos, reducción de costos, oferta de servicios y productos adecuados, que permitan proporcionar al comprador un valor similar al que proporcionan sus competidores, pero realizando actividades de modo eficaz o de una forma original que le generen mayor valor que el proporcionado por éstos.

- Finalmente, puede señalarse que el objetivo central de este documento -que buscaba analizar la incidencia de los procesos de gestión de calidad como herramienta de competitividad, específicamente para el caso del sector cooperativo- fue cumplido en la medida en que se verificaron las motivaciones y necesidades del sector en las dos principales ciudades del país, reconociendo si bien que este proceso ha llevado mejoras en las organizaciones, necesariamente no ha incidido positivamente en la competitividad de las mismas. Se requiere, entonces, un esfuerzo adicional para alcanzar que las empresas de este sector encuentren mecanismos de diferenciación y competitividad que les permitan encontrar mejores niveles de mercado y, a la vez, de productividad.

\section{Recomendaciones}

El sector cooperativo ha incrementado su participación en el mercado colombiano. Por esta razón, se ha convertido en un sector determinante en la política económica y social del país, que debe ser objeto de estudios posteriores ya que fomenta el empleo, la educación y el crecimiento económico, entre otros, bajo principios y valores claramente definidos y dentro de un ambiente participativo $y$ de asociación.

Este estudio permitió realizar un análisis de tipo descriptivo sobre la incidencia de la certificación de calidad en el sector cooperativo, un tema que no había sido sujeto de estudio anteriormente. Sin embargo, los resultados obtenidos en este documento pueden servir como base para estudios más rigurosos a futuro y con un mayor contenido estadístico, donde se puedan evaluar un mayor número de empresas. Así se podrán obtener resultados aplicables al mercado colombiano a nivel nacional y no delimitado a sólo dos ciudades.

Se podría proponer un estudio en el que, con la información financiera de estas empresas y por medio de indicadores financieros, se mediría y cuantificaría la incidencia de la certificación de calidad en los resultados económicos de la compañía y así, desde el punto de vista económico, se observarían los cambios en cuanto a crecimiento, rentabilidad y productividad.

Un estudio posterior puede enfocarse a analizar por qué la mayor participación de certificación de cooperativas se encuentra sólo en Bogotá y Medellín y por qué las otras ciudades tienen una participación tan baja; si es un fenómeno que 
obedece al hecho de que en las pequeñas ciudades la calidad no es un factor tan determinante en los procesos productivos, o si puede ser más bien producto de falta de información y conocimiento del tema o de cultura organizacional ocasionado por el miedo al cambio a nivel interno de las organizaciones.

Otro punto que podría analizarse es qué tan conveniente puede ser la normalización de los procesos y la certificación de cooperativas con respecto a acuerdos comerciales internacionales, y la implicación que puede tener con respecto al TLC.

Es este estudio, la mayor dificultad presentada fue, sin duda alguna, la recolección de la información. A pesar de que se diseñaron y usaron herramientas que facilitaron el trabajo, el nivel de abstención de las empresas fue bastante alto. Es un tema relativamente nuevo del cual no es fácil conseguir soporte teórico, ni estudios anteriores que sirvan de guía a los objetivos a conseguir.

\section{Referencias}

I. Adair, P. (1989). L'économie informelle en France: économie alternative ou société civile? En VVAA: L'autre économie: Una économie alternative? Quebec: Presses de l'Université du Quebec.

2. Buckley, J. (2000). Cómo crecer con ventaja competitiva: El valor real de la tecnologia. Santafé de Bogotá: Mc Graw Hill Interamericana S.A.

3. Consejo Nacional de Política Económica y Social. (2008). Documento CONPES 3527. Colombia.

4. Dansocial. (2006). Curso básico de economía solidaria. Bogotá: Programa de
Desarrollo Alternativo, Convenio DAPR - FIP DANSOCIAL.

5. Díaz, J.; Ballén, J.; Acosta, F. (2000). Entidades sin Ánimo de Lucro (1a ed.). Santafé de Bogotá: Legis Editores. s.A.

6. Interman Innovate Programme. (1991). Proyecto éxito e innovación en la gerencia. Colombia: cCMs/Cladea.

7. Kaoru, I. (1985). ¿Qué es el control total de calidad?: La modalidad Japonesa. Bogotá: Ediciones Norma.

8. Laville, J.L. (1994). Économie et Solidarité: esquisse d'une problématique. París: Desclée de Brouwer.

9. Levin, R.I. (1988). Estadistica para administradores ( $2^{\mathrm{a}} \mathrm{ed}$.). México: Prentice Hall Latinoamericana.

io.Mendenhall, W. \& Reinmuth, J. (1986). Estadistica para Administración y Economía. J. Díaz Saiz, F. O’ Reilly (trads.). México: Grupo Editorial Iberoamérica.

I I.Pérez Fernández de Velasco, J.A. (1994). Gestión de la calidad empresarial: Calidad en los servicios y atención al cliente: calidad total. Madrid: ESIC.

I 2.Porter, M. (1980). Competitive Strategy. New York: The Free Press.

I3.________. (2006). Estrategia y ventaja competitiva. Barcelona: Ediciones Deusto.

I4. Retolaza, J.L.; Mugarra, A. \& Enciso M. (2004). Solidarity-Based Enterprises: New Concept for the Enterprise of the Future. Segorbe: ICA Research Forum.

I 5.Revista Semana. (2009). Edición núm. 1409, 4 al 11 de mayo.

I6.Senlle, A. \& Stoll, G. (1997). Calidad total y la normalización ISO 9000: Las normas para la calidad en la práctica. Barcelona: Ediciones Gestión 200. s.A. I7.Silva, J.M. \& Dávila L. de G., R. (2000). Gestión y desarrollo: la experiencia de las cooperativas en Colombia (1 ${ }^{a}$ 
ed.). Santafé de Bogotá: Universidad Javeriana, Facultad de Estudios Ambientales y Rurales.

I 8. Superintendencia de la Economía Solidaria. (2007). Guía práctica para entidades supervisadas. Bogotá: Ministerio de Hacienda y Crédito Público.

19. Uribe, C. (1993). Curso básico de cooperativismo. Bogotá: Fondo Nacional Universitario.

\section{Normas técnicas colombianas para la certificación de empresas del sector solidario}

I. DE 296/06: Guía para la interpretación de sistemas de gestión de la calidad en organizaciones del sector solidario.

2. DE 297/06: Guía para la definición de indicadores de gestión en organizaciones del sector solidario.

3. GTC 192: Modelo de sistema de gestión para cooperativas.

4. GTC 193: Modelo de gestión para organizaciones de acción voluntaria.

5. GTC 194: Guía para la consolidación de la memoria institucional en organizaciones del sector solidario.

6. GTC 195: Sistema de información, comunicación y divulgación en las organizaciones del sector solidario.

7. GTC 196: Rendición de cuentas en organizaciones del sector solidario.

8. GTC 197: Directrices para la capacitación, formación, promoción, asistencia técnica e investigación de la base social.

9. GTC 198: Sistema de gestión de la calidad para entidades acreditadas por DANSOCIAL para impartir educación solidaria: Recomendaciones.

Io.NTC 5499: Sistema de gestión de la democracia participante en las organizaciones solidarias.

\section{Consultas en línea}

I. Alianza Cooperativa Internacional: http://www.ica.coop/

2. Alianza Cooperativa Internacional para América: www.aciamericas.coop/spip/

3. Соlлсот: www.economiasolidaria.org.

4. Confederación de Cooperativas de Colombia: www.portalcooperativo.coop/

5. CoOMEva: http://www.coomeva.com. co/archivos/multi/valores_cooperativos.pdf

6. Cooperativa lechera Colanta http:// www.colanta.com.co

7. CREDICITY Ltda.: http://www.credicity. com/Institucional/VigiladoPor/vigilado.php?seccion=in

8. Departamento Administrativo Nacional de Economía Solidaria: www.dansocial.gov.co

9. Fondo de Garantías de Entidades Cooperativas: www.fogacoop.gov.co

ıo. Instituto Colombiano de Normas Técnicas y Certificación: http://www.icontec.org

I I.Periódico Mundo Cooperativo: http:// www.mundocoop.com.ar/cooperativismo/

I 2. Revista OIKOs: http://www.economiasolidaria.net/biblioteca/A20a\%F1osRevista oikos.php

I 3. Superintendencia de la Economía Solidaria: http://www.supersolidaria.gov.co/

I 4. Superintendencia Financiera de Colombia: www.superfinanciera.gov.co 